\title{
Grain Boundary Curvature Analysis of -Grains in Ti-21S
}

\author{
D.J. Rowenhorst* , A.C. Lewis*, and G. Spanos* \\ * U.S. Naval Research Laboratory, Code 6350, Washington, DC 20375
}

The recent advances in 3D microstructural acquisition and analysis has enable the collection of larger datasets, allowing for the measurement of properties that can only be determined in 3D and evaluate them on a statistical basis. One consequence of this is that experimental results can now be directly compared to the predictions of theory. This is especially important in the process of grain growth where the topology, size, and interfacial curvatures of each individual grain in the system are required for meaningful comparisons.

This presentation will concentrate the serial-sectioning and 3-D reconstruction of -grains in Ti-21S, producing a 3-D dataset consisting of 4700 grains, as shown in Figure 1. Mechanical polishing was used to remove the material between each section resulting in a section height of $15 \mathrm{~m}$, with a total of 200 sections. Within the $2 \mathrm{D}$ plane of the sections, a high resolution image that covers a large area $(1000 \mathrm{~m} 700 \mathrm{~m})$ is created using image montaging. Alignment of the slices is achieved by using a combined ducial mark system and by minimizing the changes in the microstructure, thus preserving the long range morphological texture of the reconstruction. while achieving sub-pixel alignments from section to section. Finally, the images are segmented so that each grain is uniquely identi ed, and its properties can be measured.

By adapting the techniques outlined by MacPherson and Srolovitz [1] the curvature of the grain boundaries is determined, possible only because the full 3-D structure of the grains is known. The grain boundary curvature is related to the instantaneous growth rate of the grains (Fig. 2), and is an important parameter for understanding the microstructural evolution of the system. We nd that the grains with 1516 faces on average have a zero growth rate within the system. This is in contrast to predictions that the growth rate of grains that have near the ideal surface area to volume ratio of 13.5 faces will have a zero growth rate. We also will demonstrate how the MacPherson Srolovitz method can be adjusted so that no assumptions on the triple-junction geometry are required.

[1] R. D. MacPherson and D. J. Srolovitz, Nature, vol. 446, pp. 10531055

[2] This work was jointly sponsored by the O ce of Naval Research and DARPA as part of the Dynamic 3-D Digital Structure Program. 


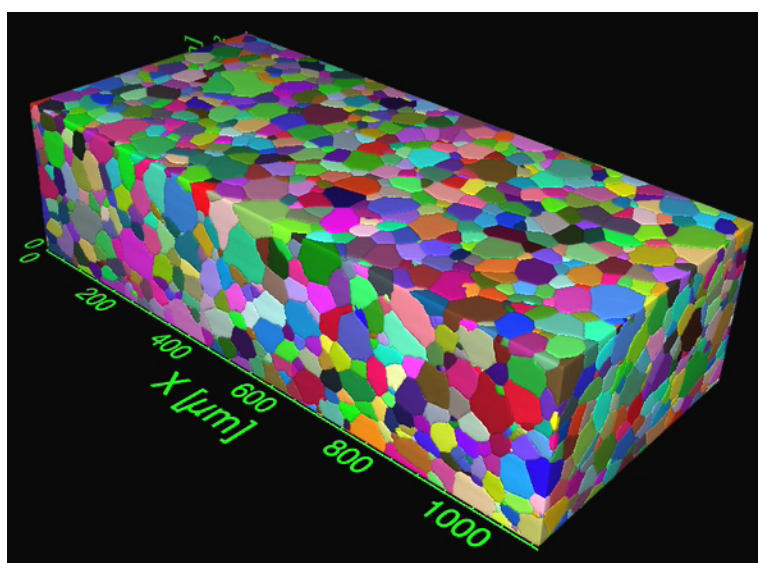

(a)

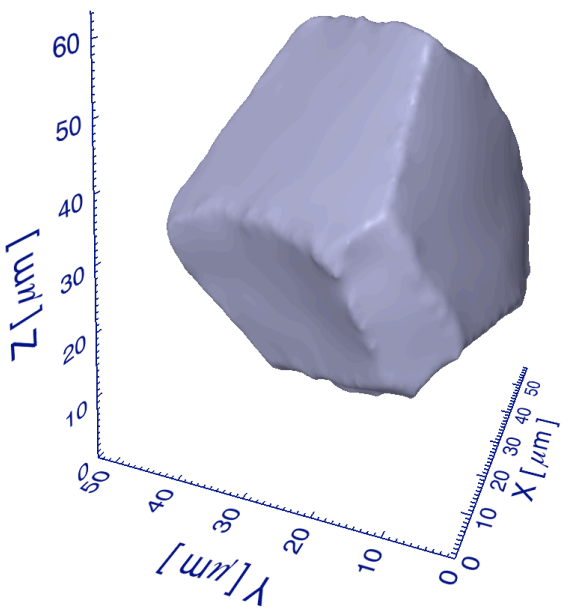

(b)

Figure 1: (a) Reconstruction of a grains within Ti-21S. The reconstruction contains over 4700 grains created by collecting over 200 serial sections. (b) One average sized grain extracted from the reconstruction.

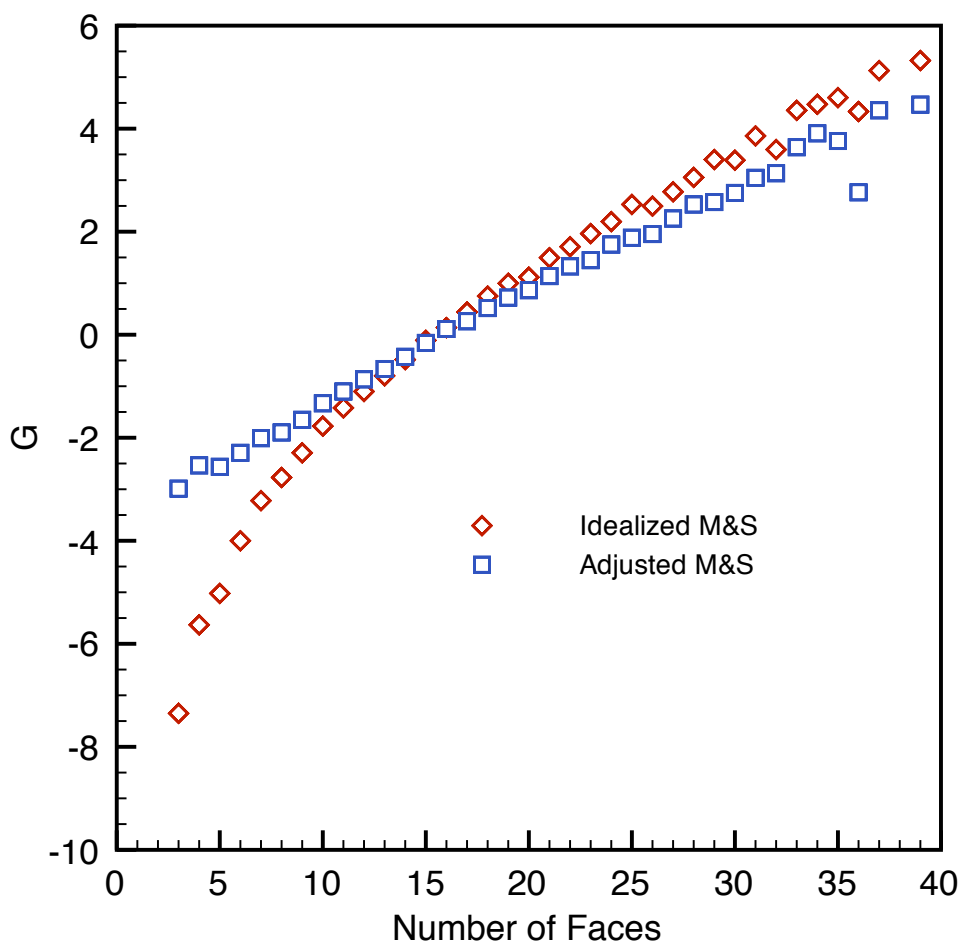

Figure 2: The normalized volumetric growth rate of the individual grains within the system. The red points indicate the rate using the idealized mean width measurement, where the dihedral angle of the triple lines is assumed to be 23 . The blue points represent the adjusted method, where no assumption is made about the geometry of the triple junctions. 